\title{
Misdiagnosis of primary pleomorphic rhabdomyosarcoma of the right thigh in a young adult: A case report
}

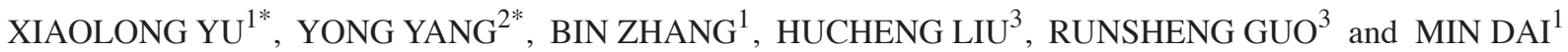 \\ ${ }^{1}$ Department of Orthopedics, The First Affiliated Hospital of Nanchang University, Artificial Joints Engineering and \\ Technology Research Center of Jiangxi Province, Nanchang, Jiangxi 330006; ${ }^{2}$ Department of Orthopedics, \\ Xinyu People's Hospital, Xinyu, Jiangxi 338025; ${ }^{3}$ Multidisciplinary Therapy Center of Musculoskeletal Tumor, \\ The First Affiliated Hospital of Nanchang University, Nanchang, Jiangxi 330006, P.R. China
}

Received March 21, 2015; Accepted April 8, 2016

DOI: $10.3892 / \mathrm{ol} .2016 .4819$

\begin{abstract}
Pleomorphic rhabdomyosarcoma (PRMS) is a rare type of soft tissue tumor accounting for $<2 \%$ of all adult sarcomas. The present study describes a case of a 28 -year-old male patient with primary PRMS of the right thigh. The patient was initially diagnosed with a schwannoma and underwent conservative therapy at a local hospital. At the 6-month follow-up, the patient reported a marked increase in the size of the mass. Finally, the patient underwent fine-needle aspiration and total tumor resection. The tumor measured $11 \times 9 \times 5 \mathrm{~cm}^{3}$ in size and was located in the vastus intermedius muscle. According to histological and immunohistochemical findings, a diagnosis of PRMS was confirmed by an expert pathology consultant. Postoperative follow-up at 3 months revealed no evidence of recurrent disease or residual side effects from therapy. However, it is imperative that such cases are closely monitored following surgery, in order to evaluate the long-term efficacy of the procedure, since misdiagnosis may increase the risk of recurrence and metastasis. The present case is noteworthy due to the misdiagnosis of PRMS, the large size of the mass and the young age of the patient.
\end{abstract}

\section{Introduction}

Rhabdomyosarcoma (RMS) is a highly malignant type of soft tissue tumor with skeletal muscle differentiation. The incidence of RMS is $\sim 43$ cases per 10 million each year for individuals

Correspondence to: Mr. Min Dai, Department of Orthopedics, The First Affiliated Hospital of Nanchang University, Artificial Joints Engineering and Technology Research Center of Jiangxi Province, 17 Yong Wai Zheng Street, Nanchang, Jiangxi 330006, P.R. China

E-mail: 1135547344@qq.com

*Contributed equally

Key words: misdiagnosis, pleomorphic rhabdomyosarcoma, young adult, right thigh, surgery, immunohistochemistry, pathology under the age of 20 (1). In patients with localized disease, the relapse-free survival rate has improved to $70-80 \%$ (2). However, the prognosis for patients with metastases is relatively poor, with a 5-year survival rate of only $30 \%$ (3). Diagnostic methods for RMS include clinical and laboratory examination, imaging analysis, pathological diagnosis and immunohistological examination (4). RMS has been divided into 3 main subtypes: Embryonal, alveolar and pleomorphic RMS (PRMS). The most common subtypes are the embryonal and alveolar subtypes (5). Primary PRMS is relatively rare and primarily affects adults, with a peak incidence in the fifth decade of life (6-9). It most commonly arises in the deep soft tissues of the extremities. Due to the similarities in clinical manifestations and imaging features between PRMS and other soft tissue tumors, PRMS is often misdiagnosed (10). The present study presents a case of PRMS that was misdiagnosed as schwannoma. This misdiagnosis resulted in the progression of the mass, and only following fine-needle aspiration and histological and immunohistochemical analysis was the tumor origin confirmed to be the skeletal muscle, and a final diagnosis of PRMS of the right thigh was provided. The present study was approved by the Ethics Review Committee of The First Affiliated Hospital of Nanchang University (Nanchang, China), and written informed consent was obtained from the patient.

\section{Case report}

In August 2014, a 28-year-old male patient presented to the Orthopedic Clinic of Taihe County People's Hospital (Ji'an, China) with a chief complaint of swelling in his right thigh for 1 month. According to the local district general hospital radiologist, magnetic resonance imaging (MRI) revealed the presence of a schwannoma and the patient was advised to undergo regular follow-up. However, 6 months later in December 2014, a gradual increase in the size of the mass and pain in the right thigh were noted. The patient was referred to the Department of Orthopedics of The First Affiliated Hospital of Nanchang University for further treatment. No personal or family history of injury or illness was recorded. A general physical examination demonstrated that the passive and active range of motion of the right knee joint was 
A

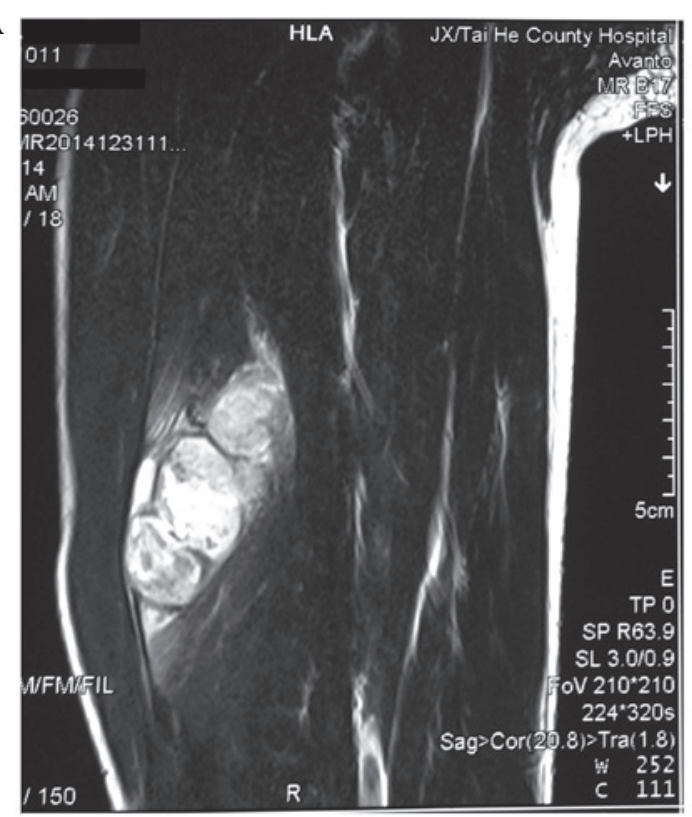

B

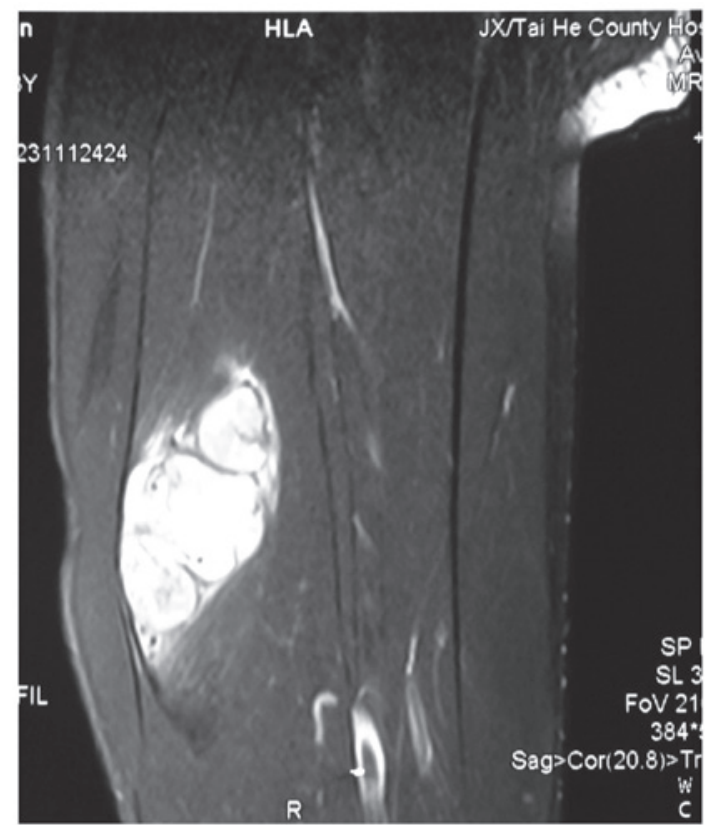

Figure 1. Preoperative magnetic resonance imaging scan. (A) Axial T2-weighted images revealed multiple cystic lesions of high-signal intensity. (B) Fat-suppressed T2-weighted images exhibited high-signal intensity.

normal, with the exception of paresthesia in the right lower extremity. No fever or respiratory embarrassment accompanied the mass, and no history of weight loss or exposure to tuberculosis was reported by the patient.

Additional physical examination revealed a poorly-defined, tender and firm soft tissue mass over the right inner thigh, but no palpable head, neck, supraclavicular, axillary or epitrochlear lymph nodes were identified. Inflammatory markers, including erythrocyte sedimentation rate and C-reactive protein, were within the normal ranges. MRI was performed for the evaluation of the mass. Axial T2-weighted images revealed multiple cystic lesions of varying sizes of high-signal intensity. Fat-suppressed T2-weighted MRI also exhibited high-signal intensity (Fig. 1A and B). In addition, fine-needle aspiration was performed to assess the mass, and cytological diagnosis was consistent with malignant neoplasm; pleomorphic spindle cell neoplasm with marked nuclear atypia and prominent mitotic activity was observed.

Based on the exclusion of surgical contraindications, surgeons affiliated with the Department of Orthopedics, The First Affiliated Hospital of Nanchang University, who specialize in treating bone tumors, performed the surgery. The patient was placed in the supine position, and following the success of epidural anesthesia, sterile drapes were disinfected and paved routinely in the right lower extremity to expose the operative field. First, a medial thigh incision was performed, $\sim 17 \mathrm{~cm}$ in length. Next, the subcutaneous tissue, superficial and deep fascia, and vastus intermedius muscle were resected layer by layer, until the femoral artery and vein were observed. The vessels above were intact. The tumor was dark brown and located in the vastus intermedius muscle. The tumor was excised completely with negative margins. Intraoperative tissue samples were extracted for pathological examination. A wound drainage tube was placed and each layer of tissue was sutured strictly following complete hemostasis. The estimated blood loss was $100 \mathrm{ml}$ and no blood transfusion was required during the procedure.

The tumor was large, smooth-surfaced and dark brown upon gross examination. The irregular tissue mass measured $\sim 11 \times 9 \times 5 \mathrm{~cm}^{3}$ in size (Fig. 2A). The resected tumor tissue was fixed in $10 \%$ formalin, embedded in paraffin and cut into $5-\mu \mathrm{m}$ sections using a microtome. The sections were subsequently stained with hematoxylin and eosin and visualized under a microscope. Microscopic examination revealed a tumor composed of interconnected bundles of atypical, spindled, pleomorphic and giant cells with high-grade atypical nuclear features. Numerous abnormal and multinucleated giant cells were observed, and the majority of cells exhibited prominent nucleoli and abundant eosinophilic cytoplasms (Fig. 2B). For immunohistochemistry, tissue sections were incubated at $25^{\circ} \mathrm{C}$ for 60 min with monoclonal mouse antibodies against desmin (catalog no., kit-0023) and myogenic differentiation 1 (MyoD1; catalog no., MAB-0119) (dilution, 1:1,000; Fuzhou Maixin Biotech Co., Ltd., Fuzhou, China). Immunohistochemical analysis revealed that the cells were positive for actin, MyoD1 and desmin, and negative for human melanoma black 45, calponin and melan-A (Fig. 2C and D). Based on these findings, a diagnosis of PRMS of the right thigh was provided.

The patient was discharged without any complications 1 week following surgery. The patient was administered 6 cycles of chemotherapy as follows: Doxorubicin, $90 \mathrm{mg} / \mathrm{day}$ for 3 days; 14 days off followed by ifosfamide $3.8 \mathrm{~g}$ /day for 5 days, followed by 14 days off prior to the next treatment cycle. At the 3-month follow up, which consisted of plain radiography and MRI, the patient was symptom-free and able to return to work. At present, the patient is currently alive and well. However, in cases like the present one, it is necessary for patients to be closely monitored, due to the high rate of recurrence and metastasis associated with misdiagnosed PRMS. 
A

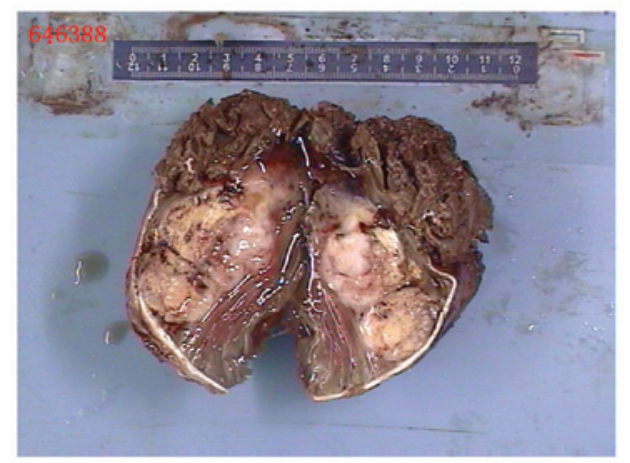

C

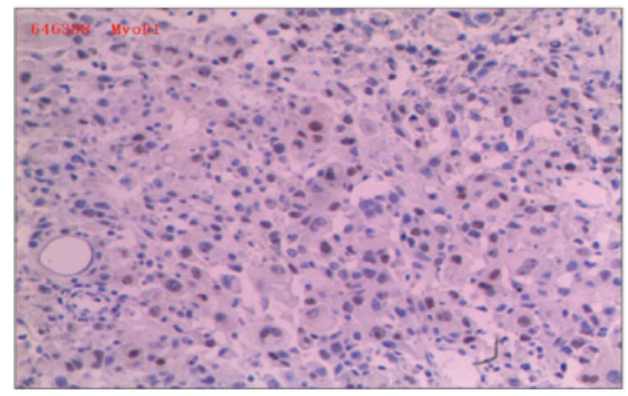

B

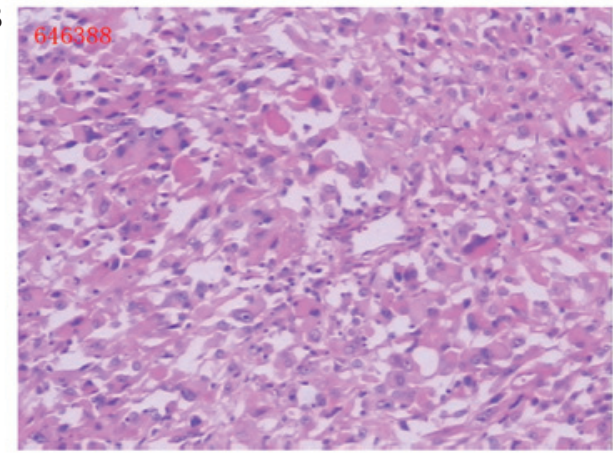

D

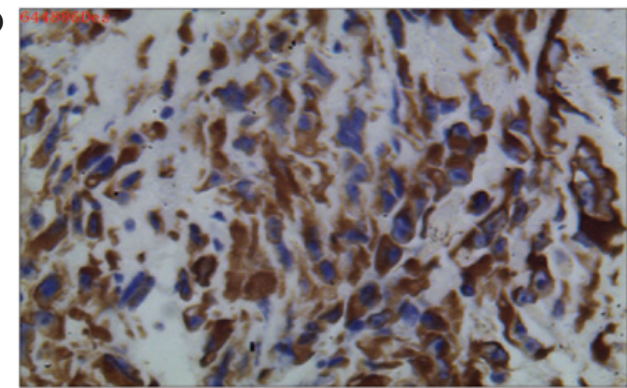

Figure 2. Pathological examination confirming pleomorphic rhabdomyosarcoma. (A) Gross appearance of the resected tumor, measuring $\sim 1 \times 9 x 5 \mathrm{~cm}^{3}$ in size. (B) Hematoxylin and eosin staining revealed spindled, pleomorphic cells arranged in a fascicular pattern. The majority of cells exhibited prominent nucleoli and abundant eosinophilic cytoplasm. (C and D) Immunohistochemical staining revealing positivity for (C) myogenic differentiation 1 and (D) desmin. (Magnification, x200).

\section{Discussion}

RMS is a highly malignant type of soft tissue tumor that arises from striated muscle cells, exhibits skeletal muscle differentiation and is associated with early and wide-spread metastasis $(11,12)$. RMS is divided into 3 main subtypes: Embryonal and alveolar RMS and PRMS, according to the 2002 World Health Organization Classification of Soft Tissue and Bone Neoplasms (5). The embryonal and alveolar subtypes are the most common, and the most frequent sites of origin of RMS include the head and neck, extremities and soft tissues. The disease has a male predominance, with a male-to-female ratio of 1.3:1 (13).

PRMS was first described by Stout in 1946 (14). Primary PRMS is relatively rare and primarily affects adults in the fifth decade of life (15). Its occurrence in young adults, as in the present case, is extremely rare. Notably, PRMS is often misdiagnosed or missed entirely, since its clinical manifestations and imaging features are similar to those of other soft tissue tumors, including fibrous histiocytoma and schwannoma (9). In the present study, the patient was misdiagnosed with schwannoma, which led to the progression of the disease for half a year; therefore, fine-needle aspiration is crucial to the diagnosis of soft tissue masses. The histological manifestations of RMS widely vary, and the histopathological diagnosis is based on morphological, immunohistochemical and ultrastructural findings that reveal a skeletal muscle phenotype $(16,17)$. PRMS is histologically distinguished from the two more common subtypes (embryonal and alveolar) by the haphazard arrangement of cells that are composed of large, pleomorphic nuclei and eosinophilic cytoplasms. PRMS cells may also be arranged in fascicles, which resembles the cell pattern observed in leiomyosarcoma (18). Histological subtyping is crucial, due to the various prognoses and therapeutic approaches used in PRMS as opposed to other soft tissue tumors. Immunohistochemistry is considered valuable for the diagnosis of PRMS, as a series of markers with a range of specificity and sensitivity is available. The primary markers of PRMS are MyoD1, desmin, sarcomeric actin and myosin $(19,20)$.

Surgical excision of PRMS is considered to be the preferred treatment, since it relieves swelling and allows for the final diagnosis to be confirmed histologically. The ideal surgical management involves complete tumor resection with negative microscopic margins (21). By contrast, RMS has particular sensitivity to external beam radiation; thus complete resection may be delayed following size reduction by radiotherapy. Certain studies have reported that RMS has an $85 \%$ overall response rate to chemotherapy $(22,23)$, in contrast to PRMS, which Ferrari et al (11) have reported a lower response rate of $6.25 \%$. PRMS predominance in adults, as well as its resistance to chemotherapy, has led to PRMS being often considered as a distinct entity from other subtypes of RMS.

In conclusion, the present case describes a 28 -year-old male patient who suffered from primary PRMS of the right thigh. Fine-needle aspiration and total tumor resection was performed, and at the 3-month follow-up, the patient had no evidence of recurrent disease or residual side effects from therapy. The performance of laboratory tests and imaging examination is particularly important in the differential diagnosis of patients presenting with soft-tissue tumors. Although the follow-up time of the current patient was relatively short, in consideration of the high risk of recurrence 
and metastasis in misdiagnosed PRMS, long-term follow-ups are often recommended in such cases.

\section{Acknowledgements}

The present study was supported by the Gan-Po Talents Project 555 of Jiangxi Province, the Support Plan of the Science and Technology Department of Jiangxi Province (grant no. 20112BBG70020) and the Natural Science Foundation of Jiangxi Province (grant no. 20132BAB205067).

\section{References}

1. Cranmer LD, Chen CC, Morgan S, Martino G and Ray J: Pleomorphic rhabdomyosarcoma in a patient with hereditary nonpolyposis colorectal cancer. J Clin Oncol 31: e108-e110, 2013.

2. Malempati S and Hawkins DS: Rhabdomyosarcoma: Review of the Children's Oncology Group (COG) Soft-Tissue Sarcoma Committee experience and rationale for current COG studies. Pediatr Blood Cancer 59: 5-10, 2012.

3. Breneman JC, Lyden E, Pappo AS, Link MP, Anderson JR, Parham DM, Qualman SJ, Wharam MD, Donaldson SS Maurer HM, et al: Prognostic factors and clinical outcomes in children and adolescents with metastatic rhabdomyosarcoma - a report from the Intergroup Rhabdomyosarcoma Study IV. J Clin Oncol 21: 78-84, 2003.

4. Scull C, Amar S, Feiz-Erfan I, Dave H and Gridley D: Adult onset primary pineal rhabdomyosarcoma. J Clin Oncol 34: e137-e40, 2016.

5. Li JJ, Forstner D and Henderson C: Cutaneous pleomorphic rhabdomyosarcoma occurring on sun damaged skin: A case report. Am J Dermatopathol 37: 653-657, 2015.

6. Stock N, Chibon F, Binh MB, Terrier P, Michels JJ, Valo I, Robin YM, Guillou L, Ranchère-Vince $\mathrm{D}$, Decouvelaere AV, et al: Adult-type rhabdomyosarcoma: Analysis of 57 cases with clinicopathologic description, identification of 3 morphologic patterns and prognosis. Am J Surg Pathol 33: 1850-1859, 2009.

7. Moretti G, Guimarães R, Oliveira KM, Sanjar F and Voegels RL: Rhabdomyosarcoma of the head and neck: 24 Cases and literature review. Braz J Otorhinolaryngol 76: 533-537, 2010 (In English, Portuguese).

8. Kefeli M, Kandemir B, Akpolat I, Yildirim A and Kokcu A: Rhabdomyosarcoma arising in a mature cystic teratoma with contralateral serous carcinoma: Case report and review of the literature. Int J Gynecol Pathol 28: 372-375, 2009.
9. Hakozaki M, Hojo H, Kuze T, Tajino T, Yamada H, Kikuta A, Qualman SJ, Kikuchi S and Abe M: Primary rhabdomyosarcoma of the sacrum: A case report and review of the literature. Skeletal Radiol 37: 683-687, 2008.

10. Mondal SK, Mandal PK, Adhikari A and Basak B: Primary pleomorphic rhabdomyosarcoma of breast: Report of a rare neoplasm. J Res Med Sci 19: 1200-1202, 2014.

11. Ferrari A, Dileo P, Casanova M, Bertulli R, Meazza C, Gandola L, Navarria P, Collini P, Gronchi A, Olmi P, et al: Rhabdomyosarcoma in adults. A retrospective analysis of 171 patients treated at a single institution. Cancer 98: 571-580, 2003.

12. Kishore B, Khare P, Gupta RJ, Gupta C and Khare V: A rare case of paratesticular pleomorphic rhabdomyosarcoma diagnosed by fine needle aspiration: A case report. Diagn Cytopathol 38: 121-126, 2010.

13. Dang ND, Teh BS and Paulino AC: Rhabdomyosarcoma arising in a previously irradiated field: An analysis of 43 patients. Int J Radiat Oncol Biol Phys 85: 598-603, 2013.

14. Stout AP: Rhabdomyosarcoma of the skeletal muscles. Ann Surg 123: 447-472, 1946

15. Fadare O, Bonvicino A, Martel M, Renshaw IL, Azodi M and Parkash V: Pleomorphic rhabdomyosarcoma of the uterine corpus: A clinicopathologic study of 4 cases and a review of the literature. Int J Gynecol Pathol 29: 122-134, 2010.

16. Caserto BG: A comparative review of canine and human rhabdomyosarcoma with emphasis on classification and pathogenesis. Vet Pathol 50: 806-826, 2013.

17. Yao JC, Wang WC, Tseng HH and Hwang WS: Primary rhabdomyosarcoma of the prostate: Diagnosis by needle biopsy and immunocytochemistry. Acta Cytol 32: 509-512, 1998.

18. Atahan S, Aksu O and Ekinci C: Cytologic diagnosis and subtyping of rhabdomyosarcoma. Cytopathology 9: 389-397, 1998.

19. Morgenstern DA, Rees H, Sebire NJ, Shipley J and Anderson J: Rhabdomyosarcoma subtyping by immunohistochemical assessment of myogenin: Tissue array study and review of the literature. Pathol Oncol Res 14: 233-238, 2008.

20. Cessna MH, Zhou H, Perkins SL, Tripp SR, Layfield L, Daines C and Coffin CM: Are myogenin and myoD1 expression specific for rhabdomyosarcoma? A study of 150 cases, with emphasis on spindle cell mimics. Am J Surg Pathol 25: 1150-1157, 2001

21. Ge X, Ma J, Dai H, Ren L, Li Q and Shi J: Clinical research on the treatment effects of radioactive (125) I seeds interstitial brachytherapy on children with primary orbital rhabdomyosarcoma. Med Oncol 31: 27, 2014.

22. Fuchs J, Dantonello TM, Blumenstock G, Kosztyla D, Klingebiel T, Leuschner I, Schuck A, Niggli FK, Koscielniak E and Seitz G: Treatment and outcome of patients suffering from perineal/perianal rhabdomyosarcoma: Results from the CWS trials-retrospective clinical study. Ann Surg 259: 1166-1172, 2014.

23. Petrović B, Djan I, Markov B, Petrović M, Erak M, Teodorović M and Baucal M: Optimal postoperative radiotherapy treatment of orbital rhabdomyosarcoma. Srp Arh Celok Lek 141: 375-379, 2013 (In Serbian). 